\title{
Matterport: Virtual Tour as A New Marketing Approach in Real Estate Business During Pandemic COVID-19
}

\author{
Mohamad Zaidi Sulaiman ${ }^{1, *}$ Mohd Nasiruddin Abdul Aziz ${ }^{2,}$ Mohd Haidar Abu \\ Bakar $^{3}$, Nur Akma Halili ${ }^{4}$, Muhammad Asri Azuddin ${ }^{5}$ \\ 1,2,4,5, Faculty of Art \& Design, Universiti Teknologi MARA, Perak Branch, 32610 Seri Iskandar, Perak, Malaysia \\ 3. Cerenkov Scientific Sdn. Bhd., 35-1, Jalan Seroja 4, Taman Seroja, 42900 Sepang, Selangor, Malaysia \\ *Corresponding author. Email: zaidi211@uitm.edu.my
}

\begin{abstract}
Real estate business was highlighted in several reports as one of the critical economic sectors affected by the pandemic COVID-19. The potential buyer cannot visit the property to experience the real space physically as before anymore. In this situation, an advance tool is needed to improve the marketing strategy in the real estate business. Currently, the 360 photography and virtual tour have become the most relevant marketing strategy to sell the property. As a new approach, Matterport, as one of the online platforms, is presented. The study engaged in an archival method or document review, thematic and content analysis. Four themes between the conventional approaches and Matterport online application were compared and discussed which are: (1) accessibility, (2) visual capture, (3) details information, (4) visual experience. This paper presents and explains the Matterport as a new platform for the marketing approach, which aims at helping the real estate business to survive during the pandemic COVID-19.
\end{abstract}

Keywords: Real Estate, 360 Photography, Virtual Tour, Matterport, Marketing.

\section{INTRODUCTION}

The process of adding value to real estate is calling real estate development. Most of the people see the development just about built a new building and subdivision only. It is referring to overall process from buying the land, designing the building, arranging the financing, obtaining public approvals, building constructing, leasing, managing and the primary purposes to selling the complete unit [1]. The real estate market is always unstable because it depends on a few factors such as interest rates, economic condition and job growth. Regarding the market behaves issues, marketing strategy is essential to manage and ensure the sales of the product.

Marketing is one of the parts in the development process that involved brokers, public relation firm, advertising agency, graphic designer, and Internet service films production [1]. The developer has to take the development risk such as leasing risk and finding a buyer or investor for the property. To get the buyer or investor, the developer will run a few marketing strategies. The marketing strategy, including creating a website, printing medium, referral encouragement, social media, brand awareness such as video form, and establish the photograph [2].

Typically, the salesman will take over the job for the next stage to serve potential client or investor to visit the property. This final process is a crucial part of getting selling the unit. Usually, the client will look for the property overall, such as the design of the building, detailing, measurement and others. It is imperative to visit the sample house to ensure everything similar to plan in agreement.

Since the outbreak pandemic COVID-19, the nature of the marketing has changed. The conventional marketing approaches in real estate business such as site visit, digital photography, 2D video slideshow, drone photo, and video has become not relevant to the current situation. Immediate relief measures need to be implemented for medium and long-term planning to reenergise the real estate and housing sector, and subsequently feeding the impact on the economy [3]. Thus, this paper intends to present an alternative approach based on practical experiences and document analysis. 


\section{LITERATURE REVIEW}

\subsection{The Impact of COVID-19 Towards Real Estate Business}

A virus spreading from Wuhan, China, namely Novel Coronavirus (2019-nCoV) was reported on January 12, 2020. The World Health Organization (WHO) verified this epidemic was affected around the world [4]. This pandemic changes everything in people live all over the world until right now. The Pandemic crisis is having a devasting effect on all economic sectors. Real estate is one of many sectors that also affected by this pandemic. The mobility restrictions and low in connectivity during COVID-19 have increased the poverty because of the higher fiscal capacity, and it was induced a segregation effect, which is inequality id higher and income per capita is lower [5]. People lost their job and sales going down drastically. The new norm of life and Movement Controlled Order (MCO) has causes limitation in real estate business to meet their client directly. This situation gives a negative impact on the marketing and selling performance.

Majority studies expected that real estate sales tended to increase in the last decade. Several studies believe that it is too early to see the impact of COVID-19 on the housing market [3]. However, certain studies show that property sales decrease drastically started in March 2020 as compared to the last year [6]. Finance research had mentioned that real estate is one of the worst sectors that affected with $72 \%$ fall dramatically [7]. Moreover, most of the countries have applied mobility restriction and blocked any investor from any foreign country.

In this research context, the mobility restriction directly affected to the conventional marketing strategy in the real-estate business. The properties tour activity on real site cannot be done because of the mobility restriction. Many conferences and meetings have changed to virtual conferences. Some brokers use the digital platform such as Skype and FaceTime to minimise the infection [3]. However, the supportive marketing approach such as digital photography, 2D video slideshow, drone photo and video enables to provide comprehensive information with real visual experience to the potential buyer. This study believes that virtual tour technology can be seen as the most relevant marketing approach in the COVID-19 pandemic for economic growth and sustainability, as suggested by the recent studies [8].

\subsection{The potential of 360 Photography \& Virtual tour}

The development of photography technology was imposing nowadays. A bulk of hardware and software were design along with the technology itself. There are a bunch of imagery technique created. Virtual reality is not a new technology. Early computerised, VR was invented in the late 1960s. Virtual Reality Photography (VRP) or known as 360 Photography is a one of technique in photography to capture the 360-degree image with borderless effect and seamless [9].

The 360 photography image usually use in virtual tour in web base to introduce or promote a location, places or space. The commercialisation of photography and online virtual environment enables marketers to utilise the virtual image technology to engage their future client as active participants via virtual reality (VR) application where they will experience the product or destination from their homes [10]. According to the Oxford English Dictionary, virtual reality refers to "computer-generated simulation of the three-dimensional image that can be interacted with in a seemingly real or physical way by a person using special electronic equipment. The viewer will experience a digital tour by using 360 photography as an interactive space. The virtual tour enables the viewer to control their point of view by themselves to point in any direction and position in the space.

In 2017, the virtual tour was very trendy among destination marketing campaigns with a creative approach from global brands. Besides, marketers assumed the positive spillover of VR experience to brand experiences [11]. This statement shows that virtual reality photography got a significant potential to be a supportive marketing tool in the real estate market. Many studies found that mental imagery or also called as the perceptual representation of nonverbal information in memory. It has been explored in marketing research as an essential mechanism of marketing stimuli process [12].

\section{CASE STUDY: MATTERPORT}

The technology of visual marketing was rapidly evolving same goes the development of new imagery technique. 2D photography and DIY video are no longer impress a new generation of buyers and investor who expect a more interactive way to experience the properties, especially during a hard time in COVID-19 pandemic. The information and visual impress fundamental key that could be provided for the buyer to understand and experience about the properties.

The landscape of the real estate business was changed. Agent and brokers who utilise the new technology of marketing will be able to engage new generation audiences [13]. New 3D virtual tour technology has recently made easy and cost-effectively to engage the next generation to experience the property.

This section presents a case study between the conventional tools and the Matterport as a new 3D virtual tour in the real-estate marketing approach. The Matterport was chosen as a single case study in this paper because it is the latest and the most popular web-based 
online software for the virtual tour. The Matterport software has been used widely in many different fields works such as conservation research [14], 3D narrowspace mapping [15], and industrial archaeology sites [16].

\subsection{Themes}

Four themes; accessibility, visual capture, details information, and visual experience were selected to be discussed based on the data from document analysis. Accessibility has become an important issue for new technology or any software applications to ensure all groups of people have the right to use and get equal benefit [17]. The visual capture capability in 3D software is affected by the characteristics, performance, and relevancy of the applications [18]. As a virtual tour application, the benefits and details information in the virtual experience affected the visit and purchasing decisions [19]. The visual experience in virtual reality platform allows the potential buyer to view the real estate products and positively impact the buyer's attitude [20].

\subsubsection{Accessibility}

Matterport is an online 3D virtual tour platform that transforms the 360 photographs into $3 \mathrm{D}$ digital interactive Twin representation of property space. The technology offers an innovative end-to-end immersive media platform that allows developers to capture and share to platform practically a 24/7 open gallery. Any potential buyer can be transported to space anytime and anywhere without any cost and commitment. Moreover, the Matterport is easy to access for the user because it is optimised for mobile (Figure 1). The application offers the developer to DIY capturing 360 photography by only using a smartphone, then upload to the Matterport web base. The rest will be processed by Matterport intelligence software to combine, realign every point of the shot and stitch them together then transform it into 3D model space. The Matterport also enables the user to share their 3D model at anywhere and anytime.

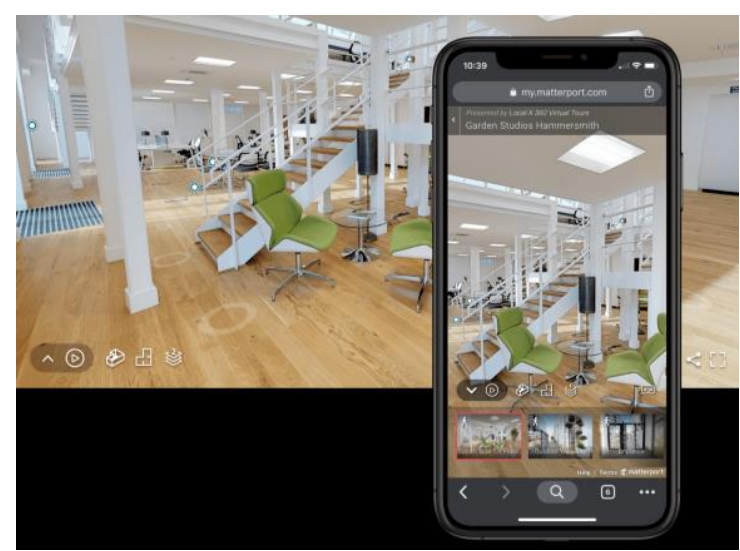

Figure 1 Matterport capture by smartphone. Retrieved September 27, 2020, from www.matterport.com

\subsubsection{Visual Capture}

The advance technology of photography in the Matterport enables the user to capture 360 photography (Figure 2). Just only with one click button, the image is already recorded in single frame compare to the conventional method which requires a professional photographer and expensive equipment. Besides that, the Matterport also capable to process image from a smartphone, which offers everyone can do the capturing process. For a better result, 360 cameras were suggested to be used to get more precise 3D model.

Aerial drones have added new dimension view of real estate in photography and video form. In advance, aerial drone's technology was innovated with the 360 photography camera which enables the viewer to explore the virtual aerial tour. With that advance technology, the more dramatic angle can be capture from the aerial view, especially to present features that are key or signature of the architecture design. However, drones technology is limited to exterior space.

Compare to the Mettaport, the visual capture is unlimited as long as the camera can fit on the location. Matterport Intelligence software is capable of stitching not only for interior but also exterior of the location.

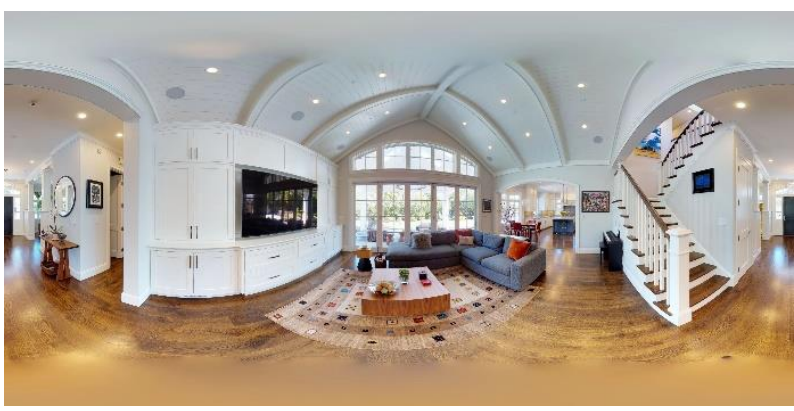

Figure 2360 Photography for Matterport virtual tours. Retrieved September 25, 2020, from www.pinterest.co.uk

\subsubsection{Details Information}

Complex information is one of the keys to pursue client purchase a property. $92 \%$ of home buyers search the internet before contacting an agent [13]. The virtual 3D image in the Mettaport included with details information such as measurement, labelling the space, and the item in every location. All that information summaries the four essential characteristics of virtual reality experience; 1 . The virtual world environment inhabited by users (including 3D digital object, space and rules); 2 . the feeling of immersion in the virtual world or actually "being there"; 3 . interactivity - the degree to 
which the user can interact with the virtual world, its objects, characters and places, and how it responds; and 4. the selective provision of sensory feedback to the user based on user input, such as navigation [21].

Compared to the conventional marketing approach, the information was minimal to mention in $2 \mathrm{D}$ form or video that make the duration of the video too long and tedious. In 3D virtual, the Matterport offers functionality such as measurement with $99 \%$ accuracy (Figure 3 ). The details information will help visitors to be more understand and experience complete information about the plan, size and design of the building before buying decision can be made.

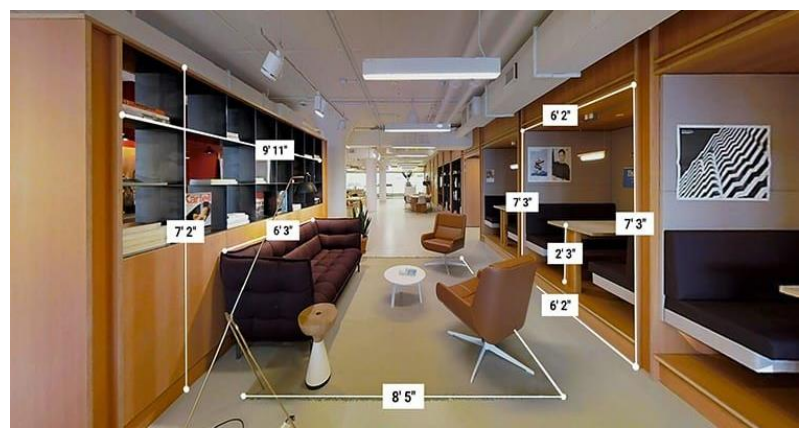

Figure 3 Accurate measurements in the Matterport. Retrieved September 28, 2020, from www.commercialobserver.com

\subsubsection{Visual Experience}

Since 1999, 360 panoramic photography was introduced and creating a new perspective of visual, which give a viewer a sense of where a scene sits with other parts of a property. There are several types of research has been done to seek and establish an empirical connection between the two main elements in virtual reality, which is mental imagery and sense of presence [10]. Prior research identified mental imagery as a process that explains the effect of website presentation on behavioural intention [21]. In the other hand, mental imagery is one of the keys in the cognitive mechanism of information with a sense of presence depending on users experience in virtual environments.

Sense of presence is the second element to complete VR. There are three essential sources of spatial cues for the formation of presence which is virtual space, physical space and mental imagery space [22]. The combination of three space will driven users attitudes and behaviours toward the virtual environments. This theory had been applied in the Matterport software that combining all the space and presented in an interactive way to achieve maximum reality tour experience (Figure 4). The combination of 2D Photography and 3D digital image in the Matterport provides a complete walkthrough experience just like 360 panoramic tours. Besides, the Mettaport more advance with the ability to take a measurement and annotate point in 3D space. Users will experience a photorealistic of the property and give them a physical presence. Compare to the video tour, the point of view is minimal. The video tour shows the viewers typically but not give them the freedom to self-tour.

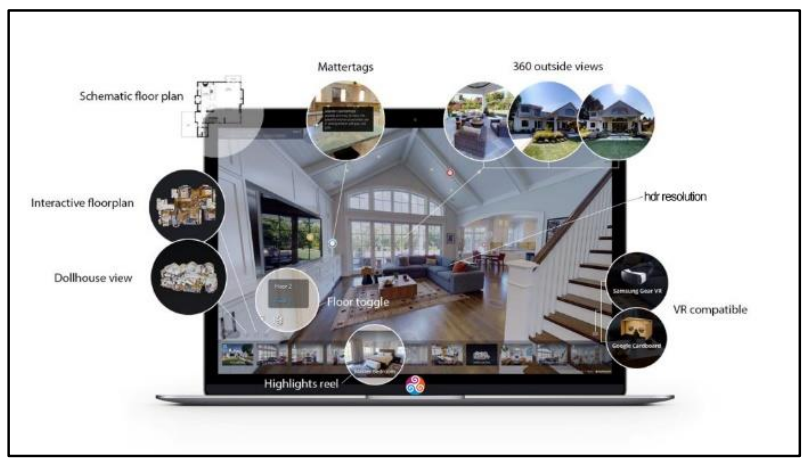

Figure 4. Matterport 3D virtual tour. Retrieved

September 25, 2020, from www.novoreperio.com

The comparison between the conventional approaches (Site visit, Digital photography, 2D Video slideshow, Drone photo \& video) and Matterport (Virtual tour) were summarised, as shown in table 1. Overall, the Matterport offers a better function with high ability as a new approach to promote the properties in the real estate business compared to the conventional approaches in terms of accessibility, visual capture, details information, and visual experience.

Table 1. Comparison between the conventional approaches with the Matterport.

\begin{tabular}{|c|c|c|}
\hline Themes & $\begin{array}{c}\text { Conventional } \\
\text { (Site Visit, Digital } \\
\text { Photography, 2D } \\
\text { Video Slideshow, } \\
\text { Drone Photo \& } \\
\text { Video) }\end{array}$ & $\begin{array}{c}\text { Matterport } \\
\text { (Virtual Tour) }\end{array}$ \\
\hline Accessibility & $\begin{array}{l}\text { - Costly } \\
\text { (equipment) } \\
\text { - Limited time } \\
\text { (site visit) }\end{array}$ & $\begin{array}{l}\text { - Mobile friendly } \\
\text {-Social sharable } \\
\text {-24 hours }\end{array}$ \\
\hline Visual capture & $\begin{array}{l}\text { - Limited for } \\
\text { interior or } \\
\text { exterior (drone) }\end{array}$ & $\begin{array}{l}\text { - Easy to DIY } \\
\text {-Interior \& Exterior }\end{array}$ \\
\hline $\begin{array}{l}\text { Details } \\
\text { Information }\end{array}$ & $\begin{array}{l}\text { - Limited } \\
\text { information }\end{array}$ & $\begin{array}{l}\text {-Measurements } \\
\text {-Labelling }\end{array}$ \\
\hline $\begin{array}{l}\text { Visual } \\
\text { Experience }\end{array}$ & $\begin{array}{l}\text { - Limited } \\
\text { viewpoint }\end{array}$ & $\begin{array}{l}\text {-Self-tour } \\
\text {-Maximum reality }\end{array}$ \\
\hline
\end{tabular}

\section{CONCLUSION}

The COVID-19 pandemic has impacted the world at a massive scale than any other disaster challenged in the last 75 years. Businesses need to come up with a new approach and strategies to sustain and minimise job losses. Mobility restriction and social distancing have reduced house views activities among potential buyers, 
which is a crucial selling process. The other conventional digital marketing strategies such as digital photography, 2D video slideshow, and drone technology has an absolute lack in terms of accessibility, visual capture, details information and visual experience.

This paper recommends the real estate industry players and house brokers to fully utilise the Matterport digital platform as a new approach in their marketing strategy because of the advance technology through 3D virtual tours. The Matterport helps to drive the decision making by the potential buyer through a hyper-real 3D virtual tour experience and completed with accurate measurements and details information for each selected item. The technology of the Matterport also proven to reduce the time and cost to the real estate business in creating building layouts and site surveys, planning remodels and upgrades, optimising emergency planning, and tracking inventory and maintenance. Overall, the Matterport technology potentially to enhance the real estate business, generate the profit, and even as lifesaving during the COVID-19 pandemic.

\section{ACKNOWLEDGMENTS}

The authors would like to express their gratitude to the Faculty of Art and Design, Universiti Teknologi MARA, Perak Branch, Seri Iskandar Campus for the research opportunity and funding support.

\section{REFERENCES}

[1] R. Peiser, "Real Estate Development," in International Encyclopedia of the Social \& Behavioral Sciences: Second Edition, Second Edi., vol. 20, Elsevier, 2015, pp. 12-19.

[2] R. Mourouzi-Sivitanidou, P. Sivitanides, R. Mourouzi-Sivitanidou, and P. Sivitanides, "Real estate economics," in Market Analysis for Real Estate, 2020, pp. 19-52.

[3] M. Nicola et al., "The socio-economic implications of the coronavirus pandemic (COVID-19): A review," Int. J. Surg., vol. 78, no. March, pp. 185-193, Jun. 2020.

[4] P. Sun, X. Lu, C. Xu, W. Sun, and B. Pan, "Understanding of COVID-19 based on current evidence," J. Med. Virol., vol. 92, no. 6, pp. 548-551, 2020.

[5] G. Bonaccorsi et al., "Economic and social consequences of human mobility restrictions under COVID-19," Proc. Natl. Acad. Sci. U. S. A., vol. 117, no. 27, pp. 15530-15535, 2020.

[6] H. Tanrıvermiş, "Possible impacts of COVID19 outbreak on real estate sector and possible changes to adopt: A situation analysis and general assessment on Turkish perspective," Journal of Urban Management, vol. 9, no. 3.
Elsevier B.V., pp. 263-269, 2020.

[7] M. Mazur, M. Dang, and M. Vega, “COVID-19 and the march 2020 stock market crash. Evidence from S\&P1500," Financ. Res. Lett., no. March, p. 101690, 2020.

[8] I. Chirisa, "Scope for Virtual Tourism in the Times of COVID-19 in Select African Destinations," J. Soc. Sci., vol. 64, no. 1-3, 2020.

[9] H. W. Wulur, S. Sentinuwo, and B. Sugiarso, "Aplikasi Virtual tour Tempat Wisata Alam di Sulawesi Utara," J. Tek. Inform., vol. 6, no. 1, pp. 1-6, 2015.

[10] V. Bogicevic, S. Seo, J. A. Kandampully, S. Q. Liu, and N. A. Rudd, "Virtual reality presence as a preamble of tourism experience: The role of mental imagery," Tour. Manag., vol. 74, no. February, pp. 55-64, 2019.

[11] T. Kim and F. Biocca, "Telepresence via Television: Two Dimensions of Telepresence May Have Different Connections to Memory and Persuasion.[1]," J. Comput. Commun., vol. 3, no. 2, pp. 0-0, 2006.

[12] M. J. Kim, C. K. Lee, and T. Jung, "Exploring Consumer Behavior in Virtual Reality Tourism Using an Extended Stimulus-OrganismResponse Model," J. Travel Res., vol. 59, no. 1, pp. 69-89, 2020.

[13] K. Stephen, "The Official Guide to Real Estate and the Blockchain," medium.com, p. 13, 2017.

[14] R. Karnicki, "Photogrammetric reconstruction software as a cost-efficient support tool in conservation research," Tech. Trans., pp. 1-13, Jul. 2020.

[15] G. Piniotis, S. Soile, F. Bourexis, M. Tsakiri, and C. Ioannidis, "Experimental Assessment Of 3d Narrow Space Mapping Technologies," ISPRS - Int. Arch. Photogramm. Remote Sens. Spat. Inf. Sci., vol. XLIII-B2-2, no. B2, pp. 149156, Aug. 2020.

[16] R. Shults et al., "Capability Of Matterport 3D Camera For Industrial Archaeology Sites Inventory," ISPRS - Int. Arch. Photogramm. Remote Sens. Spat. Inf. Sci., vol. XLII-2/W11, no. 2/W11, pp. 1059-1064, May 2019.

[17] A. Kavcic, "Software Accessibility: Recommendations and Guidelines," in EUROCON 2005 - The International Conference on "Computer as a Tool," 2005, vol. 00, pp. 1024-1027.

[18] C. Colombo, A. Del Bimbo, and A. Valli, "Visual capture and understanding of hand pointing actions in a 3-D environment," IEEE Trans. Syst. Man, Cybern. Part B Cybern., vol. 
33, no. 4, pp. 677-686, 2003.

[19] Y. Cho, Y. Wang, and R. Daniel, "Journal of Travel \& Tourism Searching for Experiences," Tourism, no. January 2012, pp. 37-41.

[20] G. Pleyers and I. Poncin, "Non-immersive virtual reality technologies in real estate: How customer experience drives attitudes toward properties and the service provider," J. Retail. Consum. Serv., vol. 57, p. 102175, 2020.

[21] S. Barnes, "Understanding Virtual Reality in Marketing: Nature, Implications and Potential," SSRN Electron. J., pp. 1-50, 2016.

[22] F. Biocca, K. Jin, and Y. Choi, "Visual touch in virtual environments: An exploratory study of presence, multimodal interfaces, and crossmodal sensory illusions," Presence Teleoperators Virtual Environ., 2001. 